\title{
Collider phenomenology of light strange-beauty squarks
}

\author{
Kingman Cheung ${ }^{1}$ and Wei-Shu Hou ${ }^{2}$ \\ ${ }^{1}$ Department of Physics and NCTS, National Tsing Hua University, Hsinchu, Taiwan, Republic of China \\ ${ }^{2}$ Department of Physics, National Taiwan University, Taipei, Taiwan, Republic of China
}

(Received 7 April 2004; published 24 August 2004)

\begin{abstract}
Strong mixing between right-handed strange and beauty squarks is a possible solution to the $C P$ violation discrepancy in $B \rightarrow \phi K_{S}$ decay as recently suggested by the Belle data. In this scenario, thanks to the strong mixing one of the strange-beauty squarks can be as light as $200 \mathrm{GeV}$, even though the generic supersymmetry scale is at TeV. In this work, we study the production of this light right-handed strange-beauty squark at hadronic colliders and discuss the detection in various decay scenarios. Detection prospect at the Tevatron run II is good for the strange-beauty squark mass up to about $300 \mathrm{GeV}$.

DOI: 10.1103/PhysRevD.70.035009

PACS number(s): 11.30.Pb, 12.15.Ff, 12.60.Jv, 14.80.Ly
\end{abstract}

\section{INTRODUCTION}

Supersymmetry (SUSY) is the leading candidate for physics beyond the standard model (SM), because it provides a weak scale solution to the gauge hierarchy problem as well as a dynamical mechanism for the electroweak symmetry breaking. Usual treatments of SUSY, however, do not address the problem of flavors. The flavor problem consists of the existence of fermion generations, their mass and mixing hierarchies, as well as the existence of $C P$ violation in quark (and now possibly also in neutrino sector) mixings, and probably has origins above the weak scale. In an interesting combination [1] of Abelian flavor symmetry (AFS) and SUSY, it was pointed out $[2,3]$ that a generic feature is the near-maximal $\tilde{s}_{R}-\widetilde{b}_{R}$ squark mixing. Such a near-maximal mixing allows for one state to be considerably lighter than the squark mass scale $\tilde{m}$. Such a state, called the strangebeauty squark $\widetilde{s b}_{1}$, carries both $s$ and $b$ flavors, and is bound to impact on $b \rightarrow s$ transitions.

It is remarkable that we may have a hint for new physics in $C P$ violation in $B \rightarrow \phi K_{S}$ decay, which is a $b \rightarrow s \bar{s} s$ transition. The SM predicts that the mixing-dependent $C P$ violation in this mode, measured in analogous way as the well established $C P$ violation in $B \rightarrow J / \psi K_{S}$ mode, should yield the same result. The Belle Collaboration, however, has found an opposite sign in the $B \rightarrow \phi K_{S}$ mode for two consecutive years $[4,5]$. The current discrepancy with SM prediction stands at a $3.5 \sigma$ level. The result from the BaBar Collaboration in 2003 is at odds [6] with Belle, but the combined result is still in $2.7 \sigma$ disagreement with $\mathrm{SM}$ expectation. While more data are needed to clarify the situation, it has been pointed out [7] that a light $\widetilde{s b}_{1}$ squark provides all the necessary ingredients to narrow this large discrepancy with SM prediction. It has (1) a large $s-b$ flavor mixing, (2) a (unique) new $C P$ violating phase, and (3) right-handed dynamics. The latter is needed for explaining why similar "wrong-sign" effects are not observed in the modes such as $B \rightarrow K_{S} \pi^{0}$ and $\eta^{\prime} K_{S}$. These modes yield consistent results as what was measured in $B \rightarrow J / \psi K_{S}$. A detailed study of various $B$ decays suggested [7] that $m_{\widetilde{s b}_{1}} \sim 200 \mathrm{GeV}$ and $m_{\tilde{g}} \sim 500 \mathrm{GeV}$ are needed, while the squark mass scale $\tilde{m}$ and other SUSY particles can be well above the TeV scale.

It is clear that a squark as light as $200 \mathrm{GeV}$ is of great interest since the Tevatron has a chance of seeing it. One should independently pursue the search for a relatively light $\widetilde{s b}_{1}$ squark, even if the $B \rightarrow \phi K_{S} C P$ violation discrepancy evaporates in the next few years. We note that a strangebeauty squark, carrying $\sim 50 \%$ in strange and beauty flavor, would lead to a weakening of bounds on beauty squark search based on $b$-tagging. In this work, we study direct strange-beauty squark-pair production, as well as the feed down from gluino-pair production and the associated production of $\widetilde{s b}_{1}$ with a gluino. It turns out that the dominant contribution comes from direct squark-pair production as long as the squark mass is below $300 \mathrm{GeV}$. However, for squark mass above $300 \mathrm{GeV}$, the feed down from gluino-pair production with $m_{\tilde{g}}=500 \mathrm{GeV}$ becomes important. We also study various decay scenarios of the strange-beauty squarks at the Tevatron, which is of immediate interest. The most interesting decay mode is $\widetilde{s b}_{1} \rightarrow b / s+\widetilde{\chi}_{1}^{0}$, which gives rise to $a$ final state of multi- $b$ jets plus large missing energies. The other scenarios considered are the $\widetilde{s b}$ - $\mathrm{LSP}$ and the $R$-parity violating $\widetilde{s b}_{1}$ decay possibilities.

The organization of the paper is as follows. In Sec. II we recapitulate the features of the model needed for our collider study. We discuss the production of the strange-beauty squark at hadronic machines in Sec. III, and its decay modes and detection in Sec. IV. Conclusion is given in Sec. V.

\section{INTERACTIONS}

We do not go into the details of the model, but mention that the $d$ flavor is decoupled [3] to evade the most stringent low energy constraints. The generic class of AFS models $[1,2]$ imply a near-maximal $s_{R}-b_{R}$ mixing, which is extended to the right-handed squark sector upon invoking SUSY. We focus only on the $2 \times 2$ right-handed strange and beauty squarks, which are strongly mixed. The mass matrix is given by

$$
\mathcal{L}=-\left(\tilde{s}_{R}^{*} \tilde{b}_{R}^{*}\right)\left(\begin{array}{ll}
\tilde{m}_{22}^{2} & \tilde{m}_{23}^{2} e^{-i \sigma} \\
\tilde{m}_{23}^{2} e^{i \sigma} & \tilde{m}_{33}^{2}
\end{array}\right)\left(\begin{array}{c}
\tilde{s}_{R} \\
\tilde{b}_{R}
\end{array}\right) .
$$


Since the mass matrix is hermitian and the phase freedom has already been used for quarks, so there remains only one $C P$ violating phase $[2,3,7]$. However, for collider studies it is not yet relevant. With the transformation

$$
\left(\begin{array}{c}
\tilde{s}_{R} \\
\widetilde{b}_{R}
\end{array}\right)=R\left(\begin{array}{l}
\widetilde{s b}_{1} \\
\widetilde{s b}_{2}
\end{array}\right)=\left(\begin{array}{ll}
\cos \theta_{m} & \sin \theta_{m} \\
-\sin \theta_{m} e^{i \sigma} & \cos \theta_{m} e^{i \sigma}
\end{array}\right)\left(\begin{array}{l}
\widetilde{s b}_{1} \\
\widetilde{s b}_{2}
\end{array}\right),
$$

the mass term is diagonalized as

$$
\mathcal{L}=-\left(\widetilde{s b}_{1}^{*} \widetilde{s b}_{2}^{*}\right)\left(\begin{array}{ll}
\tilde{m}_{1}^{2} & 0 \\
0 & \tilde{m}_{2}^{2}
\end{array}\right)\left(\begin{array}{l}
\widetilde{s b}_{1} \\
\widetilde{s b}_{2}
\end{array}\right) .
$$

The diagonalization matrix $R$ enters the gluino-quarksquark and squark-squark-gluon interactions. Assuming the quarks are already in mass eigenbasis, the relevant gluinoquark-squark interaction in the mass eigenbasis is

$$
\begin{aligned}
\mathcal{L}= & -\sqrt{2} g_{s} T_{k j}^{a}\left[-\overline{\tilde{g}_{a}} P_{R} s_{j} \widetilde{s b}_{1 k}^{*} \cos \theta_{m}\right. \\
& +{\overline{\sigma_{a}}}_{P_{R}} b_{j} \widetilde{s b}_{1 k}^{*} \sin \theta_{m} e^{-i \sigma}-\overline{\widetilde{g}_{a}} P_{R} s_{j} \widetilde{s b}_{2 k}^{*} \sin \theta_{m} \\
& \left.-\overline{\widetilde{g}}_{a} P_{R} b_{j} \widetilde{s b}_{2 k}^{*} \cos \theta_{m} e^{-i \sigma}+\text { H.c. }\right],
\end{aligned}
$$

where $P_{R}=\left(1+\gamma^{5}\right) / 2$, and $a, j, k$ are the color indices for gluinos, quarks and squarks, respectively. The squarksquark-gluon interaction is

$$
\begin{aligned}
\mathcal{L}= & -i g_{s} A_{\mu}^{a} T_{i j}^{a}\left(\widetilde{s b}_{1 i}^{*} \overleftrightarrow{\partial}_{\mu} \widetilde{s b}_{1 j}+\widetilde{s b}_{2 i}^{*} \stackrel{\leftrightarrow}{\partial}_{\mu} \widetilde{s b}_{2 j}\right) \\
& +g_{s}^{2}\left(T^{a} T^{b}\right)_{i j} A^{a \mu} A_{\mu}^{b}\left(\widetilde{s b}_{1 i}^{*} \widetilde{s b}_{1 j}+\widetilde{s b}_{2 i}^{*} \widetilde{s b}_{2 j}\right),
\end{aligned}
$$

where

$$
\left(T^{a} T^{b}\right)_{i j}=\frac{1}{6} \delta_{a b} \delta_{i j}+\frac{1}{2}\left(d_{a b c}+i f_{a b c}\right) T_{i j}^{c} .
$$

The relevant Feynman rules are listed in Fig. 1.

\section{PRODUCTION AT HADRONIC MACHINES}

We have set the generic SUSY scale at TeV, except for the gluino and the light strange-beauty squark $\widetilde{s b_{1}}$, which could be as light as 500 and $200 \mathrm{GeV}$, respectively. These masses are still allowed by the squark-gluino search at the Tevatron [8]. In fact, these limits are more forgiving for the present case because $\widetilde{s b}$ does not decay into $b$ quark $100 \%$ of the time.

\section{A. Processes and formulas}

The production of the strange-beauty squark can proceed via the following processes.

(1) $q \bar{q}$ and $g g$ fusion [Figs. 2(a) and 2(b)]

$$
q \bar{q}, g g \rightarrow \widetilde{s b_{1}} \widetilde{s b}_{1}^{*} .
$$

If the initial state is $s \bar{s}$ or $b \bar{b}$, there is an additional
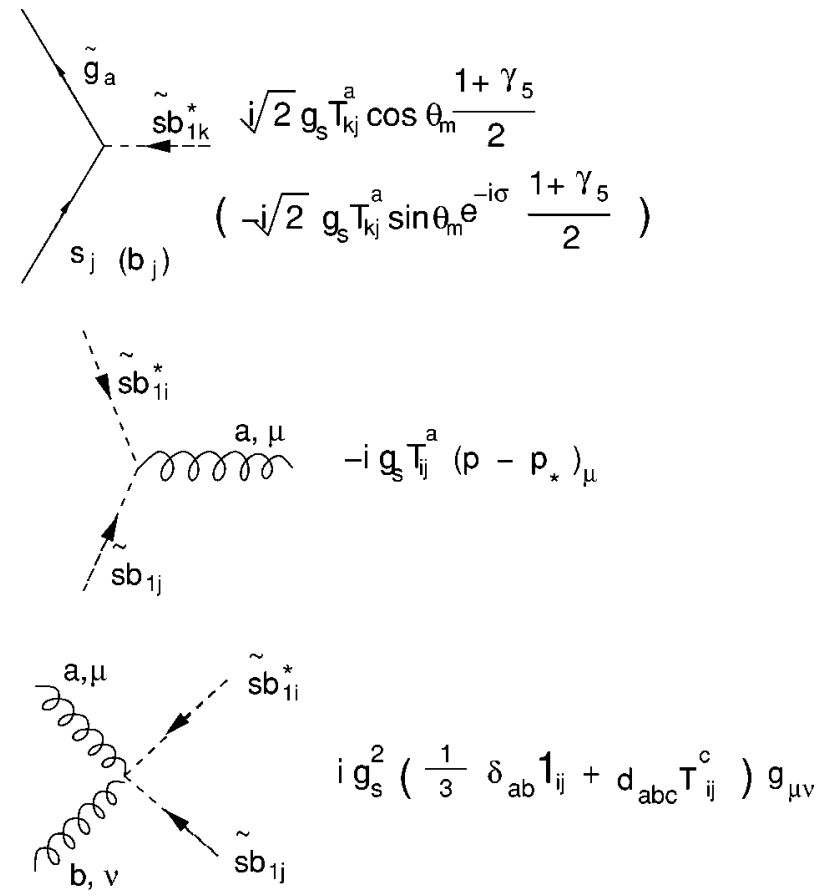

FIG. 1. The relevant Feynman rules used in this work. The momenta are going into the vertex.

contribution from the $t$-channel gluino exchange diagram, shown in Fig. 2(c). Note that there are also $s \bar{b}, b \bar{s} \rightarrow \widetilde{s b}_{1} \widetilde{s b}_{1}^{*}$ contributions via the $t$-channel gluino exchange diagram only.

(2) The $s s, b b, \bar{s} \bar{s}, \bar{b} \bar{b}, s b, \bar{s} \bar{b}$ initial state scattering via $t$ - and $u$-channel gluino exchange diagrams

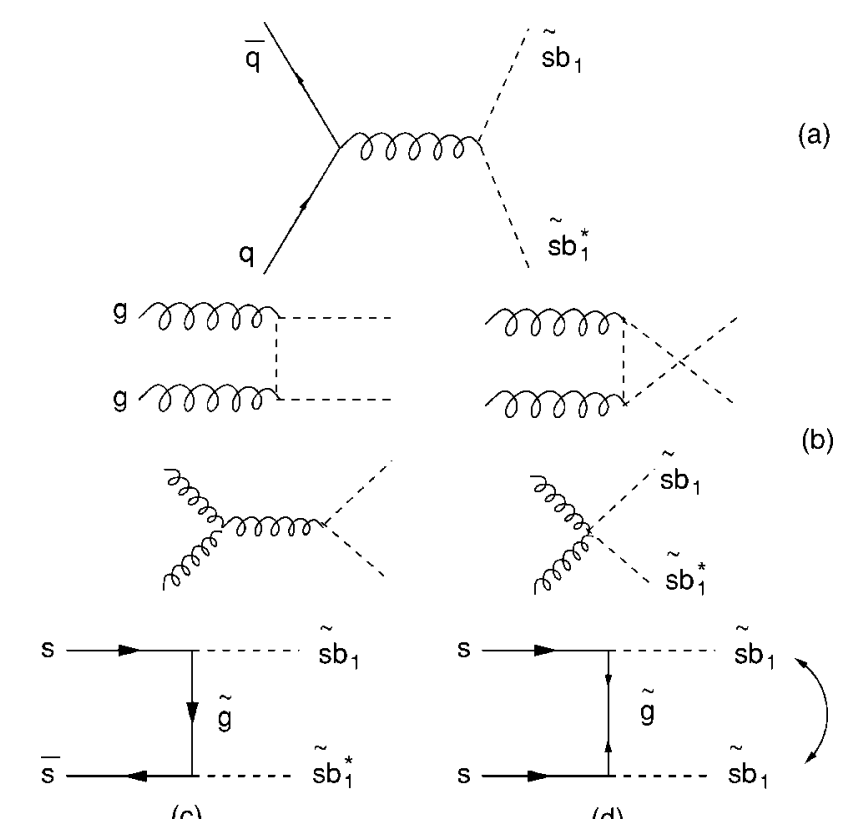

(c)

(d)

FIG. 2. Contributing Feynman diagrams for (a) $q \bar{q} \rightarrow \widetilde{s b}_{1} \widetilde{s b}_{1}^{*}$, (b) $g g \rightarrow \widetilde{s b}_{1} \widetilde{s b}_{1}^{*}, \quad$ (c) $s \bar{s}(b \bar{b}) \rightarrow \widetilde{s b}_{1} \widetilde{s b}_{1}^{*}$, and (d) $s s(b b)$ $\rightarrow \widetilde{s b}_{1} \widetilde{g b}_{1}$. 
$s s, s b, b b \rightarrow \widetilde{s b_{1}} \widetilde{s b}_{1}, \quad \bar{s} \bar{s}, \bar{s} \bar{b}, \bar{b} \bar{b} \rightarrow \widetilde{s b}_{1}^{*} \widetilde{s b}_{1}^{*}$,

shown in Fig. 2(d).

(3) Gluino pair production, followed by gluino decay,

$$
q \bar{q}, g g \rightarrow \tilde{g} g ; \quad \tilde{g} \rightarrow s \widetilde{s b}_{1}^{*}, b \widetilde{s b}_{1}^{*}, \widetilde{s s b_{1}}, \overrightarrow{b s b_{1}} .
$$

For $s \bar{s}, b \bar{b}$ in the initial states there are additional $t$ - and $u$-channel diagrams. Note that $s \bar{b}, \bar{s} b \rightarrow \tilde{g} \tilde{g}$ are also possible through the $t$ - and $u$-channel diagrams.

(4) Associated production of $\widetilde{s b}_{1}$ with gluino

$$
s g, b g \rightarrow \widetilde{s b} \tilde{g},
$$

followed by gluino decay.

Since the gluino has a mass of at least $500 \mathrm{GeV}$, we expect the $t$-or $u$-channel gluino-exchange diagrams to be much smaller than $q \bar{q}$ annihilation diagrams. Moreover, the $t$-or $u$-channel gluino-exchange diagrams are only relevant for $s$ or $b$ in the initial state, so the contributions of which are further suppressed by their parton luminosities. Nevertheless, we include all those $t$-channel gluino diagrams when the initial state quarks are $s$ or $b$. In gluino-pair production we also keep the $t$ - and $u$-channel $\widetilde{s b}_{1}$-exchange diagrams for the initial state quarks $s$ or $b$.

\section{Direct production of $\widetilde{s b}_{1} \widetilde{s b}_{1}^{*}$}

Let us first introduce some short-hand notation. The $\hat{s}, \hat{t}, \hat{u}$ are the usual Mandelstem variables. We define the following

$$
\begin{aligned}
& \hat{t}_{\tilde{g}}=\hat{t}-m_{\tilde{g}}^{2}, \quad \hat{u}_{\tilde{g}}=\hat{u}-m_{\tilde{g}}^{2}, \\
& \hat{t}_{s b}=\hat{t}-m_{\widetilde{s b_{1}}}^{2}, \quad \hat{u}_{s b}=\hat{u}-m_{\widetilde{s b_{1}}}^{2}, \\
& \beta_{s b}=\sqrt{1-\frac{4 m_{\widetilde{s b}_{1}}^{2}}{\hat{s}}, \quad \beta_{g}=\sqrt{1-\frac{4 m_{\tilde{g}}^{2}}{\hat{s}}},} \\
& \beta_{s b g}=\sqrt{\left(1-\frac{m_{\tilde{g}}^{2}}{\hat{s}}-\frac{m_{\widetilde{s b}}^{2}}{\hat{s}}\right)^{2}-4 \frac{m_{\tilde{g}}^{2}}{\hat{s}} \frac{m_{\widetilde{s b}}^{2}}{\hat{s}}} .
\end{aligned}
$$

The subprocess cross section for $q \bar{q} \rightarrow \widetilde{s b}_{1} \widetilde{s b}_{1}^{*}$ is given by

$$
\begin{aligned}
& \frac{d \sigma}{d \cos \theta^{*}}\left(q \bar{q} \rightarrow \widetilde{s b}_{1} \widetilde{s b}_{1}^{*}\right) \\
& \quad=\frac{2 \pi \alpha_{s}^{2}}{9 \hat{s}} \beta_{s b}\left[\frac{1}{4}\left(1-\beta_{s b}^{2} \cos ^{2} \theta^{*}\right)-\frac{m_{\widetilde{s b}}^{2}}{\hat{s}}\right],
\end{aligned}
$$

where $\theta^{*}$ is the central scattering angle in the parton rest frame. Integrating over the scattering angle $\theta^{*}$, the cross section is given by

$$
\sigma\left(q \bar{q} \rightarrow \widetilde{s b}_{1} \widetilde{s b}_{1}^{*}\right)=\frac{2 \pi \alpha_{s}^{2}}{27 \hat{s}} \beta_{s b}^{3} .
$$

The differential cross section for $g g \rightarrow \widetilde{s b}_{1} \widetilde{s b}_{1}^{*}$ is

$$
\begin{aligned}
& \frac{d \sigma}{d \cos \theta^{*}}\left(g g \rightarrow \widetilde{s b_{1}} \widetilde{s b}_{1}^{*}\right) \\
& \quad=\frac{\pi \alpha_{s}^{2}}{256 \hat{s}} \beta_{s b}\left(\frac{64}{3}-\frac{48 \hat{u}_{s b} \hat{t}_{s b}}{\hat{s}^{2}}\right)\left(1-\frac{2 \hat{s}_{\widetilde{s b}_{1}}^{2}}{\hat{u}_{s b} \hat{t}_{s b}}+\frac{2 \hat{s}^{2} m_{s b_{1}}^{4}}{\hat{u}_{s b}^{2} \hat{t}_{s b}^{2}}\right) .
\end{aligned}
$$

The integrated cross section is given by

$$
\begin{aligned}
\sigma\left(g g \rightarrow \widetilde{s b}_{1} \widetilde{s b}_{1}^{*}\right)= & \frac{\pi \alpha_{s}^{2}}{\hat{s}}\left[\beta_{s b} \frac{5 \hat{s}+62 m_{\widetilde{s b_{1}}}^{2}}{48 \hat{s}}\right. \\
& \left.+\frac{m_{\widetilde{s b}_{1}}^{2}}{6 \hat{s}} \frac{m_{\widetilde{s b}_{1}}^{2}+4 \hat{s}}{\hat{s}} \ln \frac{1-\beta_{s b}}{1+\beta_{s b}}\right] .
\end{aligned}
$$

For completeness we also give the expressions for $s \bar{s}, b \bar{b}$ $\rightarrow \widetilde{s b_{1}} \widetilde{s b}_{1}^{*}$ cross sections,

$$
\begin{aligned}
& \frac{d \sigma}{d \cos \theta^{*}}\left(s \bar{s} \rightarrow \widetilde{s b}_{1} \widetilde{s b}_{1}^{*}\right) \\
& =\frac{2 \pi \alpha_{s}^{2} \beta_{s b}}{9 \hat{s}}\left(\frac{1}{4}\left(1-\beta_{s b}^{2} \cos ^{2} \theta^{*}\right)-\frac{m_{\widetilde{s b}}^{2}}{\hat{s}}\right) \\
& \quad \times\left[1-\frac{1}{3} \frac{\hat{s}}{\hat{t}_{\tilde{g}}} \cos ^{2} \theta_{m}+\frac{1}{2} \frac{\hat{s}^{2}}{\hat{t}_{\tilde{g}}^{2}} \cos ^{4} \theta_{m}\right] .
\end{aligned}
$$

Integrating over $\cos \theta^{*}$ gives

$$
\begin{aligned}
\sigma(s \bar{s} \rightarrow & \left.\widetilde{s b}_{1} \widetilde{s b}_{1}^{*}\right)=\frac{2 \pi \alpha_{s}^{2}}{27 \hat{s}^{3}}\left\{\beta _ { s b } \hat { s } \left(\hat{s} \beta_{s b}^{2}-6 \hat{s} \cos ^{4} \theta_{m}\right.\right. \\
& \left.+\cos ^{2} \theta_{m}\left(\hat{s}+2 m_{-}^{2}\right)\right)+\cos ^{2} \theta_{m}\left(2 m_{-}^{4}-3 \hat{s} \cos ^{2} \theta_{m}\right. \\
& \left.\left.\times\left(\hat{s}+2 m_{-}^{2}\right)+2 \hat{s} m_{\tilde{g}}^{2}\right) \log \left(\frac{\hat{s}+2 m_{-}^{2}-\beta_{s b} \hat{s}}{\hat{s}+2 m_{-}^{2}+\beta_{s b} \hat{s}}\right)\right\},
\end{aligned}
$$

where $m_{-}^{2}=m_{\tilde{g}}^{2}-m_{\widetilde{s b_{1}}}^{2}$. The cross section for $b \bar{b} \rightarrow \widetilde{s b}_{1} \widetilde{s b}_{1}^{*}$ can be obtained by replacing $\cos ^{2} \theta_{m} \leftrightarrow \sin ^{2} \theta_{m}$ in Eqs. (14) and (15). On the other hand, the processes $s \bar{b}, b \bar{s} \rightarrow \widetilde{s b}_{1} \widetilde{s b}_{1}^{*}$ only have the $t$-channel gluino exchange diagram, and its differential cross section is given by

$$
\begin{aligned}
\frac{d \sigma}{d \cos \theta^{*}}\left(s \bar{b} \rightarrow \widetilde{s b}_{1} \widetilde{s b}_{1}^{*}\right)= & \frac{\pi \alpha_{s}^{2} \beta_{s b}}{9} \frac{\hat{s}}{\hat{t}_{\tilde{g}}^{2}} \cos ^{2} \theta_{m} \sin ^{2} \theta_{m} \\
& \times\left(\frac{1}{4}\left(1-\beta_{s b}^{2} \cos ^{2} \theta^{*}\right)-\frac{m_{\widetilde{s b}_{1}}^{2}}{\hat{s}}\right) .
\end{aligned}
$$




\section{Direct production of $\widetilde{s b}_{1} \widetilde{s b}_{1}$}

Production of $\widetilde{s b}_{1} \widetilde{s b}_{1}\left(\widetilde{s b}_{1}^{*} \widetilde{s b}_{1}^{*}\right)$ pair requires $s s, s b$ or $b b(\overline{s s}, \bar{s} \bar{b}$, or $\bar{b} \bar{b})$ in the initial state. The process proceeds via $t$ - and $u$-channel gluino-exchange diagrams, as shown in Fig. 2(d). The differential cross section is given by

$$
\begin{aligned}
& \frac{d \sigma}{d \cos \theta^{*}}\left(s s \rightarrow \widetilde{s b}_{1} \widetilde{s b}_{1}\right) \\
& \quad=\frac{\pi \alpha_{s}^{2} \beta_{s b}}{18} \cos ^{4} \theta_{m} m_{\tilde{g}}^{2}\left[\frac{1}{\hat{t}_{\tilde{g}}^{2}}+\frac{1}{\hat{u}_{\tilde{g}}^{2}}-\frac{2}{3} \frac{1}{\hat{t}_{\tilde{g}}} \frac{1}{\hat{u}_{\tilde{g}}}\right],
\end{aligned}
$$

where we have explicitly put in the factor $1 / 2$ and so $\cos \theta^{*}$ ranges from -1 to 1 . Integrating over the angle the total cross section is

$$
\begin{aligned}
\sigma\left(s s \rightarrow \widetilde{s b}_{1} \widetilde{s b}_{1}\right)= & \frac{\pi \alpha_{s}^{2} \beta_{s b}}{18} \cos ^{4} \theta_{m} m_{\tilde{g}}^{2}\left[\frac{4}{m_{-}^{4}+\hat{s} m_{\tilde{g}}^{2}}\right. \\
& \left.+\frac{8}{3 \beta_{s b} \hat{s}} \frac{1}{\hat{s}+2 m_{-}^{2}} \log \left(\frac{\hat{s}+2 m_{-}^{2}-\beta_{s b} \hat{s}}{\hat{s}+2 m_{-}^{2}+\beta_{s b} \hat{s}}\right)\right] .
\end{aligned}
$$

The cross section for $b b \rightarrow \widetilde{s b} \widetilde{s b}_{1}$ can be obtained by replacing $\cos ^{4} \theta_{m} \leftrightarrow \sin ^{4} \theta_{m}$, while that for $s b \rightarrow \widetilde{s b}_{1} \widetilde{s b}_{1}$ by replacing $\cos ^{4} \theta_{m} \leftrightarrow \cos ^{2} \theta_{m} \sin ^{2} \theta_{m}$. Note that, for example, the amplitude of $b b \rightarrow \widetilde{s b_{1}} \widetilde{s b}_{1}$ contains the phase factor $e^{-2 i \sigma}$. Obviously, when we calculate the cross section the phase factor drops out.

\section{Feed down from gluino-pair production}

We employ a tree-level calculation for gluino-pair production, though including the next-to-leading order (NLO) corrections [9] the cross section may increase by more than $50 \%$. However, the overall gluino-pair production is small because we have chosen the gluino mass to be at least 500 $\mathrm{GeV}$. Whether we include the NLO correction or not does not affect our conclusion.

Here we give the tree-level formulas for gluino-pair production, without the squark in the $t$ - and $u$-channels,

$$
\begin{aligned}
\frac{d \sigma}{d \cos \theta^{*}}(q \bar{q} \rightarrow \tilde{g} \tilde{g})= & \frac{2 \pi \alpha_{s}^{2}}{3 \hat{s}} \beta_{g} \frac{\hat{t}_{\tilde{g}}^{2}+\hat{u}_{\tilde{g}}^{2}+2 m_{\tilde{g}}^{2} \hat{s}}{\hat{s}^{2}}, \\
\frac{d \sigma}{d \cos \theta^{*}}(g g \rightarrow \tilde{g} \tilde{g})= & \frac{9 \pi \alpha_{s}^{2}}{16 \hat{s}} \beta_{g}\left(1-\frac{\hat{t}_{\tilde{g}} \hat{u}_{\tilde{g}}}{\hat{s}^{2}}\right)\left(\frac{\hat{s}^{2}}{\hat{t}_{\tilde{g}} \hat{u}_{\tilde{g}}^{\tilde{g}}}\right. \\
& \left.-2+\frac{4 m_{\tilde{g}}^{2} \hat{s}}{\hat{t}_{\tilde{g}} \hat{u}_{\tilde{g}}}-\frac{4 \hat{s}^{2} m_{\tilde{g}}^{4}}{\hat{t}_{\tilde{g}}^{2} \hat{u}_{\tilde{g}}^{2}}\right),
\end{aligned}
$$

where we have put in the factor of $1 / 2$ for identical particles in the final state, and $\cos \theta^{*}$ is from -1 to 1 . The integrated cross sections are given by

$$
\begin{aligned}
\sigma(q \bar{q} \rightarrow \tilde{g} \tilde{g})= & \frac{8 \pi \alpha_{s}^{2}}{9 \hat{s}} \beta_{g}\left(1+\frac{2 m_{\tilde{g}}^{2}}{\hat{s}}\right), \\
\sigma(g g \rightarrow \tilde{g} \tilde{g})= & \frac{3 \pi \alpha_{s}^{2}}{4 \hat{s}}\left[-\beta_{g}\left(4+17 \frac{m_{\tilde{g}}^{2}}{\hat{s}}\right)\right. \\
& \left.+3\left(\frac{4 m_{\tilde{g}}^{4}}{\hat{s}^{2}}-\frac{4 m_{\tilde{g}}^{2}}{\hat{s}}-1\right) \log \left(\frac{1-\beta_{g}}{1+\beta_{g}}\right)\right] .
\end{aligned}
$$

For completeness we also give the cross sections for $s \bar{s}$ $\rightarrow \tilde{g} \tilde{g}$

$$
\begin{aligned}
\frac{d \sigma}{d \cos \theta^{*}}(s \bar{s} \rightarrow \tilde{g} \tilde{g})= & \frac{2 \pi \alpha_{s}^{2}}{3 \hat{s}} \beta_{g}\left\{\frac{\hat{t}_{\tilde{g}}^{2}+\hat{u}_{\tilde{g}}^{2}+2 m_{\tilde{g}}^{2} \hat{s}}{\hat{s}^{2}}\right. \\
& +\frac{2}{9} \cos ^{4} \theta_{m}\left(\frac{\hat{t}_{\tilde{g}}^{2}}{\hat{t}_{s b}^{2}}+\frac{\hat{u}_{\tilde{g}}^{2}}{\hat{u}_{s b}^{2}}\right) \\
& +\frac{1}{2} \cos ^{2} \theta_{m} \frac{1}{\hat{s}}\left(\frac{\hat{s} m_{\tilde{g}}^{2}+\hat{t}_{\tilde{g}}^{2}}{\hat{t}_{s b}}+\frac{\hat{s} m_{\tilde{g}}^{2}+\hat{u}_{\tilde{g}}^{2}}{\hat{u}_{s b}}\right) \\
& \left.+\frac{1}{18} \cos ^{4} \theta_{m} \frac{\hat{s} m_{\tilde{g}}^{2}}{\hat{u}_{s b} \hat{t}_{s b}}\right\}
\end{aligned}
$$

The formulas for $b \bar{b} \rightarrow \tilde{g} \tilde{g}$ can be obtained by replacing $\cos \theta_{m}$ by $\sin \theta_{m}$. Note that $s \bar{b}, \bar{s} b \rightarrow \widetilde{g} \tilde{g}$ only occur via the $t$ and $u$-channel diagrams, and the differential cross section is given by

$$
\begin{aligned}
\frac{d \sigma}{d \cos \theta^{*}}(s \bar{b} \rightarrow \tilde{g} \tilde{g})= & \frac{\pi \alpha_{s}^{2}}{27 \hat{s}} \beta_{g} \cos ^{2} \theta_{m} \sin ^{2} \theta_{m}\left\{4\left(\frac{\hat{t}_{\tilde{g}}^{2}}{\hat{t}_{s b}^{2}}+\frac{\hat{u}_{\tilde{g}}^{2}}{\hat{u}_{s b}^{2}}\right)\right. \\
& \left.+\frac{\hat{s} m_{\tilde{g}}^{2}}{\hat{u}_{s b} \hat{t}_{s b}}\right\} .
\end{aligned}
$$

We have chosen the mass of gluino to be at least $500 \mathrm{GeV}$, in order not to upset lower energy constraints such as $b$ $\rightarrow s \gamma$ rate, and not to violate the bound from direct search at the Tevatron [8]. The gluino so produced will decay into a strange or beauty quark plus the strange-beauty squark $\widetilde{s b}_{1}$. Therefore, gluino-pair production gives two more jets in the final state than direct production. Having more jet activities to tag on may help the detection, especially when $b$-tagging is employed. We shall discuss in more detail in the next section when we treat the decay of the $\widetilde{s b}_{1}$. Nevertheless, since the gluino mass is above $500 \mathrm{GeV}$, the production rate at the Tevatron is rather small. For a gluino mass of 500 $\mathrm{GeV}$, the production cross section is $2.9 \mathrm{fb}$, which may increase to about $4 \mathrm{fb}$ after taking into account NLO correction [9]. However, it helps only a little as far as the strange- 

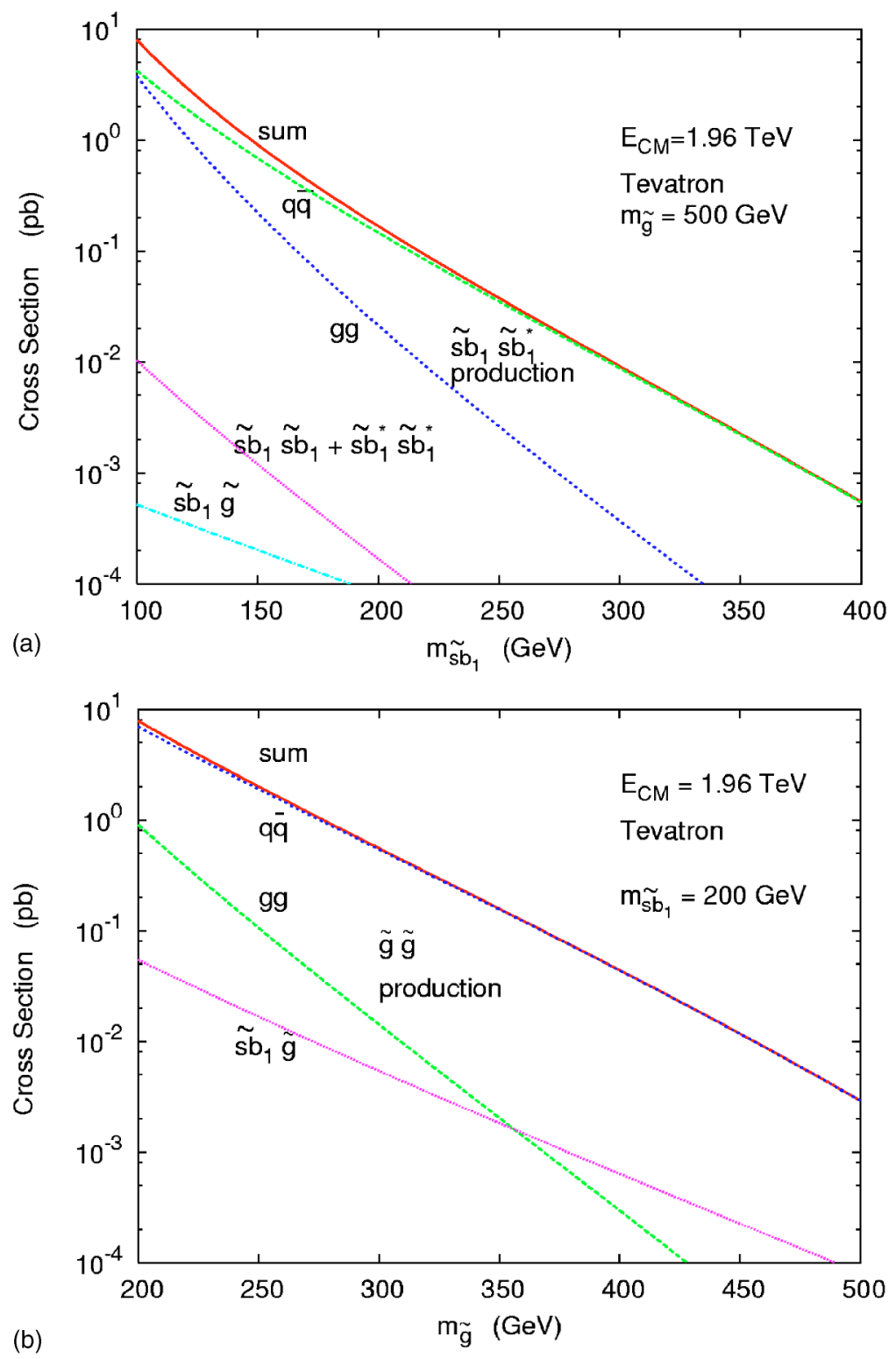

FIG. 3. Total cross section for direct production of (a) the $\widetilde{s b_{1}} \widetilde{s b}_{1}^{*}$ pair and (b) the $\tilde{g} \tilde{g}$ pair at the Tevatron. The individual $g g$ fusion and $q \bar{q}$ annihilation contributions are shown. In (a) we also show $\widetilde{s b}_{1} \widetilde{s b}_{1}+\widetilde{s b}_{1}^{*} \widetilde{s b}_{1}^{*}$ production, and $\widetilde{s b}_{1} \tilde{g}+\widetilde{s b}_{1}^{*} \tilde{g}$ production, where we have fixed $m_{g}=500 \mathrm{GeV}$. In (b) we also show $\widetilde{s b}_{1} \tilde{g}$ $+\widetilde{s b}_{1}^{*} \tilde{g}$ production, where we have fixed $m_{\widetilde{s b}_{1}}$ $=200 \mathrm{GeV}$. beauty squark pair production is concerned, unless the squark mass is above $300 \mathrm{GeV}$. We will take this into account in our analysis.

\section{Production of $\widetilde{s b} \tilde{g}$}

There is another process $s(b) g \rightarrow \widetilde{g} \widetilde{s b}$ that can contribute to strange-beauty squark production, but it requires either $s$ or $b$ in the initial state. The differential cross section for the process is given by

$$
\begin{aligned}
\frac{d \sigma}{d \cos \theta^{*}}(s g \rightarrow & \left.\widetilde{s b}_{1} \tilde{g}\right) \\
= & \frac{\pi \alpha_{s}^{2}}{192 \hat{s}} \beta_{s b g} \cos ^{2} \theta_{m}\left[24\left(1-\frac{2 \hat{s} \hat{u}_{s b}}{\hat{t}_{\tilde{g}}^{2}}\right)-\frac{8}{3}\right]\left[-\frac{\hat{t}_{\tilde{g}}}{\hat{s}}\right. \\
& \left.+\frac{2\left(m_{\tilde{g}}^{2}-m_{\widetilde{s b}_{1}}^{2}\right) \hat{t}_{\tilde{g}}}{\hat{s} \hat{u}_{s b}}\left(1+\frac{m_{\widetilde{s b}_{1}}^{2}}{\hat{u}_{s b}}+\frac{m_{\tilde{g}}^{2}}{\hat{t}_{\tilde{g}}}\right)\right] .
\end{aligned}
$$

For the $b g$ initial state, the above formula is modified by changing $\cos ^{2} \theta_{m} \leftrightarrow \sin ^{2} \theta_{m}$.

\section{B. Production cross sections}

Folding in parton distributions with the parton level cross sections, the cross sections for direct $\widetilde{s b} \widetilde{s b}_{1}^{*}$ pair production at the Tevatron are shown in Fig. 3(a), where we give the individual $g g$ and $q \bar{q}$ contributions. As expected, the gluon fusion contribution is subdominant for $m_{\widetilde{s b}} \gtrsim 100 \mathrm{GeV}$. We also show in Fig. 3(a) the same sign $\widetilde{s b}_{1} \widetilde{s b}_{1}+\widetilde{s b}_{1}^{*} \widetilde{s b}_{1}^{*}$ production, and the associated $\widetilde{s b}_{1} \tilde{g}+\widetilde{s b}_{1}^{*} \tilde{g}$ production. These processes are three orders of magnitude smaller than $\widetilde{s b}_{1} \widetilde{s b}_{1}^{*}$ pair production, and can be safely ignored at the Tevatron.

Gluino-pair production cross sections at the Tevatron are given in Fig. 3(b). Similar to squark-pair production, gluinopair production is dominated by $q \bar{q}$ pair annihilation. For a 

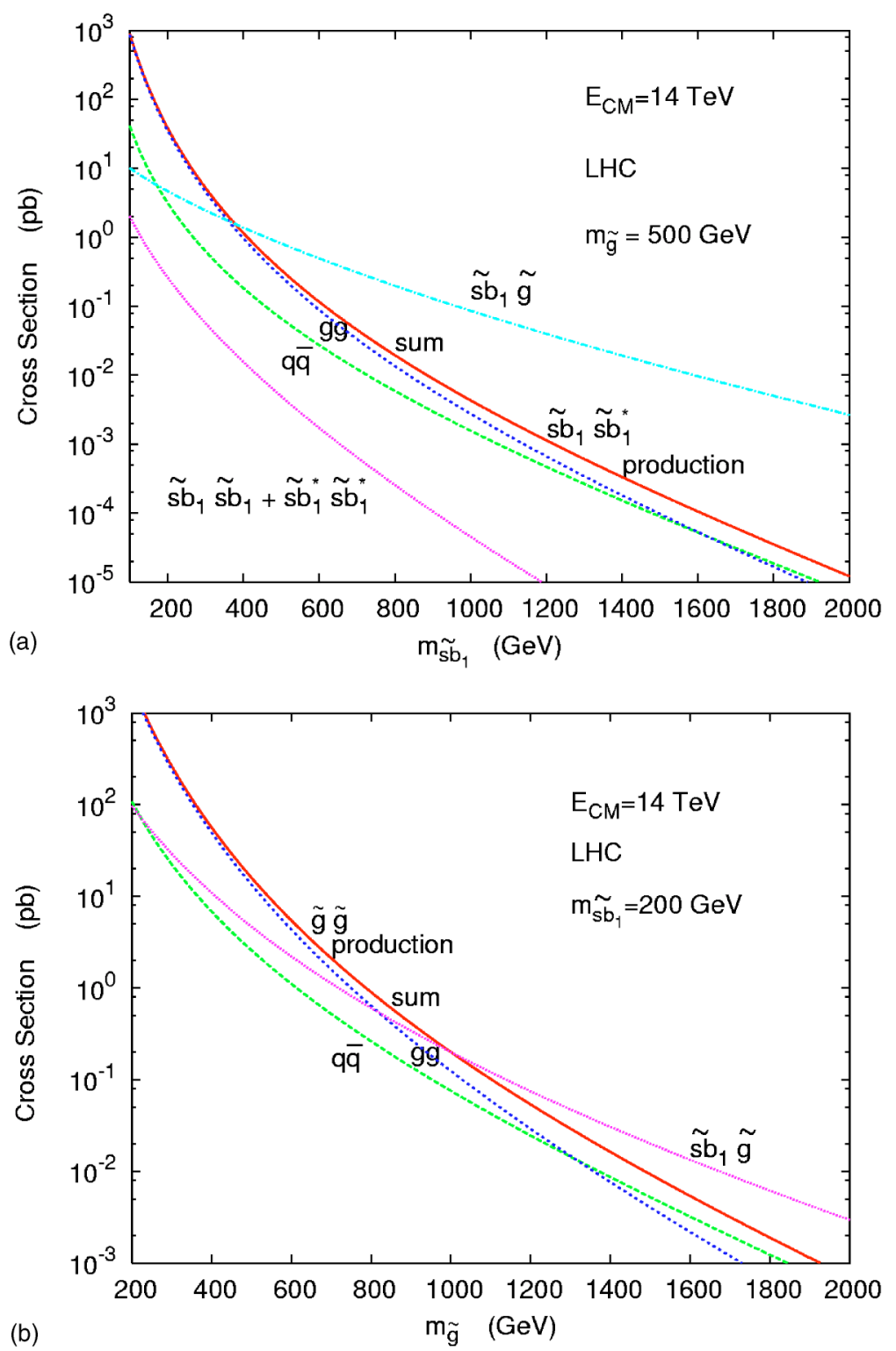

FIG. 4. Total cross section for direct production of (a) the $\widetilde{s b_{1}} \widetilde{s b}_{1}^{*}$ pair and (b) the $\tilde{g} \tilde{g}$ pair at the LHC. The individual $g g$ fusion and $q \bar{q}$ annihilation contributions are shown. In (a) we also show $\widetilde{s b}_{1} \widetilde{s b}_{1}+\widetilde{s b}_{1}^{*} \widetilde{s b}_{1}^{*}$ production, and $\widetilde{s b}_{1} \tilde{g}$ $+\widetilde{s b}_{1}^{*} \tilde{g}$ production, where we have fixed $m_{\tilde{g}}$ $=500 \mathrm{GeV}$. In (b) we also show $\widetilde{s b}_{1} \widetilde{g}+\widetilde{s b}_{1}^{*} \tilde{g}$ production, where we have fixed $m_{\widetilde{s b}}$ $=200 \mathrm{GeV}$. gluino mass of $500 \mathrm{GeV}$ the cross section is only a few fb, and thus this contribution becomes comparable to direct $\widetilde{s b}_{1}$ pair production only when $m_{\widetilde{s b}_{1}} \gtrsim 300 \mathrm{GeV}$. Therefore, at low $m_{\widetilde{s b}_{1}}$ the gluino contribution is very small, while at high $m \widetilde{s b}_{1}$ the gluino contribution can 0extend the sensitivity further.

The situation is different at the LHC. We show the corresponding results in Figs. 4(a) and 4(b), respectively. We see that gluon fusion now dominates over $q \bar{q}$ pair annihilation. Furthermore, gluino pair production and squark pair production cross sections are both above $10 \mathrm{pb}$ for $m_{\widetilde{s b}_{1}}$ $=200 \mathrm{GeV}$ and $m_{g}=500 \mathrm{GeV}$, and both contributions have to be taken into account at the LHC. We also show the associated $\widetilde{s b}_{1} \tilde{g}+\widetilde{s b}_{1}^{*} \tilde{g}$ production cross section in Figs. 4(a) and 4(b). These curves are somewhat misleading, however, that their cross sections become larger than $\widetilde{s b}_{1}$-pair (or gluinopair) production for large enough $m_{\widetilde{s b}_{1}}\left(m_{\tilde{g}}\right)$. This is simply because the mass of $m_{g}$ is held fixed at $500 \mathrm{GeV}$ in Fig. 4(a) while $m_{s b_{1}}$ is fixed at $200 \mathrm{GeV}$ in Fig. 4(b). Therefore, for very large mass the $\widetilde{s b}_{1}$-or $\tilde{g}$-pair production become suppressed.

Before we discuss detection, we need to understand how the $\widetilde{s b}_{1}$ squark decays, to which we now turn.

\section{DECAY AND DETECTION OF THE STRANGE- BEAUTY SQUARK}

If the SUSY scale is set at TeV, all SUSY particles should be around this scale, unless one has cancellation mechanisms in the diagonalization of the neutralino, chargino, or sfermion mass matrices that allow some of them to become close to the electroweak scale. The lightness of the $\widetilde{s b}_{1}$ in our scenario is a particular example of this type. This in fact involves fine-tuning. However, the fine-tuning is comparable [3] to what is already seen in the quark mixing matrix. In any case, we do not discuss it further here. 
We concentrate on squark-pair production at the Tevatron. We put the LHC study aside as its discussion is more intricate, but of less immediate interest. It is clear from Fig. 3(a) that the dominant production channels are $g g, q q$ $\rightarrow \widetilde{s b_{1}} \widetilde{s b}_{1}^{*}$. Gluino-pair production with $m_{g}=500 \mathrm{GeV}$, followed by gluino decay, is only relevant for $m_{\widetilde{s b}}$ $\gtrsim 300 \mathrm{GeV}$. On the other hand, $\widetilde{s b}_{1} \widetilde{s b}_{1}$ and $\widetilde{s b}_{1}^{*} \widetilde{s b}_{1}^{*}$ pair production and the associated production can be safely ignored.

In the following, we take on three situations for the decay of the strange-beauty squark:

(i) When the $\widetilde{s b}_{1}$ is the lightest supersymmetric particle (LSP) and $R$-parity is conserved. ${ }^{1}$ This stable $\widetilde{s b}_{1}$ case also includes the case when the $\widetilde{s b}_{1}$ is stable within the detector but decays outside.

(ii) The $\widetilde{s b}_{1}$ is the LSP but $R$-parity is violated such that it will decay into 2 jets or 1 lepton plus 1 jet.

(iii) The $\widetilde{s b}_{1}$ is the next-to-lightest supersymmetric particle (NLSP), and either neutralino (in supergravity) or gravitino (gauge-mediated) is the LSP such that $\widetilde{s b}_{1}$ will decay into a strange or beauty quark plus the neutralino or gravitino.

Among the three cases we particularly emphasize case (iii), which is the most popular. In the SUGRA models, one has $\widetilde{s b}_{1} \rightarrow s / b \widetilde{\chi}_{1}^{0}$, while in gauge-mediated models $\widetilde{s b}_{1}$ $\rightarrow s / b \widetilde{G}$ or $\widetilde{s b}_{1} \rightarrow s / b \widetilde{\chi}_{1}^{0} \rightarrow s / b \quad \gamma \widetilde{G}$. In any case, there will be $b / s$-quark jets plus a large missing energy in the final state. We simplify the picture by modelling the decay as $\widetilde{s b}_{1}$ $\rightarrow s / b \tilde{\chi}_{1}^{0}$ and by varying the mass of the neutralino.

\section{A. Stable strange-beauty squark}

In this case, the $\widetilde{s b}_{1} \widetilde{s b}_{1}^{*}$ pair so produced will hadronize into color-neutral hadrons by combining with some light quarks. Such objects are strongly-interacting massive particles, electrically either neutral or charged. If the hadron is electrically neutral, it will pass through the tracker with little trace. The interactions in the calorimetry would be rather intricate, since charge exchange $(\bar{d}$ replaced by $\bar{u}$ when

\footnotetext{
${ }^{1} \mathrm{~A}$ concern of this squark-LSP scenario is that the dark matter cannot be a colored or charged particle. However, one has to actually calculate the relic density of the LSP. Since the squarks annihilate into SM particles via strong interaction, the relic density, which scales inversely with the annihilation cross section, is in fact very small, as long as the squark mass is less than $1-2 \mathrm{TeV}$. Therefore, such a squark-LSP scenario is safe and consistent with cosmological constraints. A similar study on gluino-LSP scenario was performed in Ref. [13]. In these cases, the dark matter has to be some other particle or substance, which might be viewed as a drawback of such pictures, but in principle they are possible.
}

passing by a nucleus) can readily occur. ${ }^{2}$ However, the hadron could be electrically charged with equal probability. In this case, the hadron will undergo ionization energy loss in the central tracking system, hence behaves like a "heavy muon." Let us discuss this possibility since it is more straightforward.

The energy loss $d E / d x$ due to ionization in the detector material is very standard [10]. Essentially, the penetrating particle loses energy by exciting the electrons of the material. Ionization energy loss $d E / d x$ is a function of $\beta \gamma \equiv p / M$ and the charge $Q$ of the penetrating particle. The dependence on the mass $M$ of the penetrating particle comes in through $\beta \gamma$ for a large mass $M$ and small $\gamma$ [10]. In other words, $d E / d x$ is the same for different masses if the $\beta \gamma$ values of these particles are the same. For the range of $\beta \gamma$ between 0.1 and 1 that we are interested in, $d E / d x$ has almost no explicit dependence on the mass $M$ of the penetrating particle. Therefore, when $d E / d x$ is measured in an experiment, the $\beta \gamma$ can be deduced, which then gives the mass of the particle if the momentum $p$ is also measured. Hence, $d E / d x$ is a good tool for particle identification for massive stable charged particles. In fact, the CDF Collaboration has made a few searches for massive stable charged particles [11]. The CDF analyses required that the particle produces a track in the central tracking chamber and/or the silicon vertex detector, and at the same time penetrates to the outer muon chamber. ${ }^{3}$

The CDF detector has a silicon vertex detector and a central tracking chamber (which has a slightly better resolution in this regard), which can measure the energy loss $(d E / d x)$ of a particle via ionization, especially at low $\beta \gamma<0.85$ ( $\beta$ $<0.65$ ) where $d E / d x \sim 1 / \beta^{2}$. Once the $d E / d x$ is measured, the mass $M$ of the particle can be determined if the momentum $p$ is measured simultaneously. Furthermore, the particle is required to penetrate through the detector material and make it to the outer muon chamber, provided that it has an initial $\beta>0.25-0.45$ depending on the mass of the particle [11]. Therefore, the CDF requirement on $\beta$ or $\beta \gamma$ is (note $\left.\beta \gamma=\beta / \sqrt{1-\beta^{2}}\right)$

$$
0.25-0.45 \lesssim \beta<0.65 \Leftrightarrow 0.26-0.50 \lesssim \beta \gamma<0.86 \text {. }
$$

The lower limit is to make sure that the penetrating particle can make it to the outer muon chamber, while the upper limit makes sure that the ionization loss in the tracking chamber is sufficient for detection. CDF has searched for such massive stable charged particles, but did not find any. The limits placed on the mass of these particles are model dependent [12]. Some theoretical studies on massive stable charged par-

\footnotetext{
${ }^{2} \mathrm{An}$ issue arises when the neutral hadron containing the $\widetilde{s b}_{1}$ may "bounce" into a charged hadron when the internal $\bar{d}$ is knocked off and replaced by a $\bar{u}$, for example. The probability of such a scattering depends crucially on the mass spectrum of the hadrons formed by $\widetilde{s b}_{1}$. In reality, we know very little about the spectrum, so we simply assume a $50 \%$ chance that a $\widetilde{s b}$, will hadronize into a neutral or charged hadron.

${ }^{3}$ In run II, the requirement to reach the outer muon chamber may be dropped but it leads to a lower signal-to-background ratio.
} 


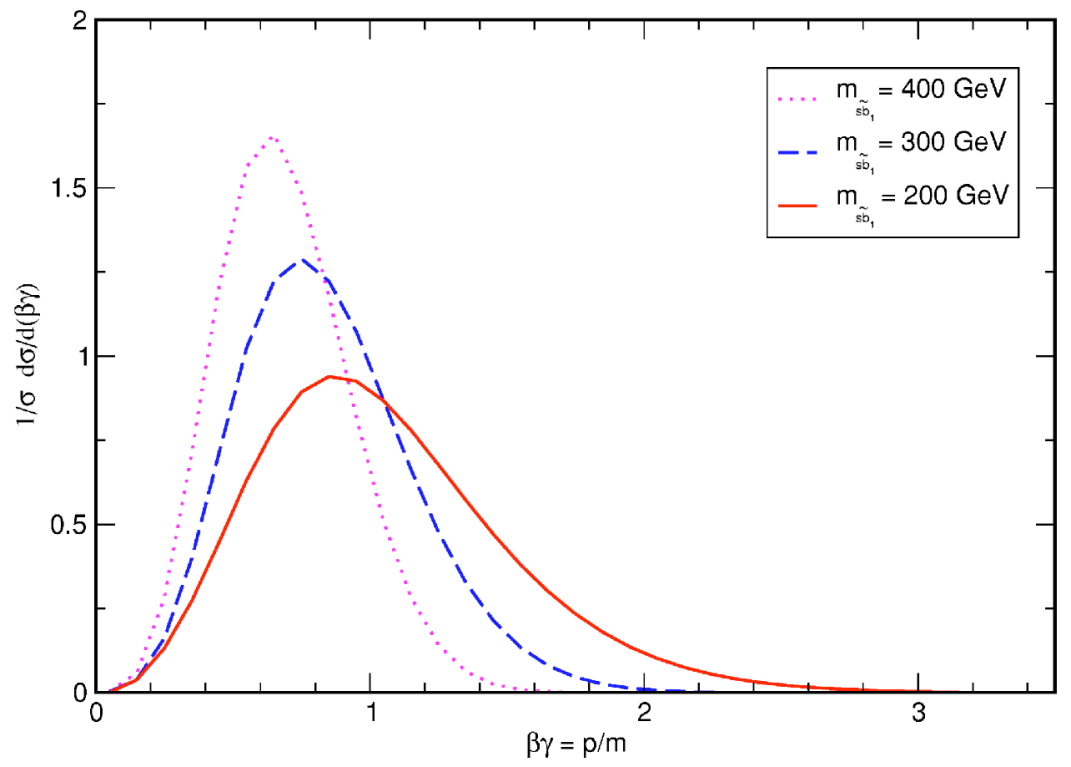

FIG. 5. The $\beta \gamma \equiv p / m_{\widetilde{s} b_{1}}$ spectrum for squarkpair production at the Tevatron, where $p$ is the squark momentum. ticles exist for gluino LSP models [13], colored Higgs bosons and Higgsinos [14], and scalar leptons [15].

We use a similar analysis for strange-beauty squark pair production with the squark remaining stable within the detector. We employ the following acceptance cuts on the squarks

$$
p_{T}\left(\widetilde{s b}_{1}\right)>20 \mathrm{GeV}, \quad\left|y\left(\widetilde{s b}_{1}\right)\right|<2.0, \quad 0.25<\beta \gamma<0.85
$$

In Fig. 5, we show the $\beta \gamma$ distribution for direct $\widetilde{s b}_{1}$ pair production at the Tevatron. It is clear that more than half of the cross sections satisfy the $\beta \gamma$ cut. This is easy to understand as the squark is massive such that they are produced close to threshold. In Table I we show the cross sections from direct $\widetilde{s b}_{1}$ pair production with all the acceptance cuts in Eq. (24), for detecting 1 massive stable charged particle (MCP), 2 MCPs, or at least 1 MCPs in the final state. The latter cross section is the simple sum of the former two. We have used a probability of $50 \%$ that the $\widetilde{s b}_{1}$ will hadronize into a charged hadron. In the table, we also give the feed down from gluino-pair production in the parentheses. It is

TABLE I. Cross sections for direct strange-beauty squark pair production at the Tevatron, with the cuts of Eq. (24). Here $\sigma_{1 \mathrm{MCP}}$, $\sigma_{2 \mathrm{MCP}}$ denote requiring the detection of 1,2 massive stable charged particles (MCP) in the final state, respectively. Requiring at least one MCP in the final state corresponds to simply adding the two cross sections. In parentheses, we give the contribution fed down from direct gluino-pair production.

\begin{tabular}{cccc}
\hline \hline$m_{\widetilde{s b}_{1}}(\mathrm{GeV})$ & $\sigma_{1 \mathrm{MCP}}(\mathrm{fb})$ & $\sigma_{2 \mathrm{MCP}}(\mathrm{fb})$ & $\sigma_{\geqslant 1 \mathrm{MCP}}(\mathrm{fb})$ \\
\hline 200 & $41(0.46)$ & $9.3(0.02)$ & $50(0.48)$ \\
250 & $10.9(0.96)$ & $2.8(0.14)$ & $14(1.1)$ \\
300 & $3.1(1.2)$ & $0.91(0.3)$ & $4.0(1.5)$ \\
350 & $0.87(1.3)$ & $0.29(0.43)$ & $1.2(1.8)$ \\
400 & $0.23(1.4)$ & $0.088(0.48)$ & $0.32(1.8)$ \\
450 & $0.058(1.4)$ & $0.024(0.51)$ & $0.082(1.9)$ \\
\hline \hline
\end{tabular}

obvious that the feed down is relatively small for $m_{\widetilde{s b}_{1}}$ $\lesssim 300 \mathrm{GeV}$, but becomes significant for $m_{\widetilde{s b}} \gtrsim 300 \mathrm{GeV}$. Requiring about 10 such events as suggestive evidence, the sensitivity can reach up to almost $m_{\widetilde{s b}_{1}} \simeq 300 \mathrm{GeV}$ with an integrated luminosity of $2 \mathrm{fb}^{-1}$.

\section{B. $\widetilde{s b}_{1}$ as LSP but $R$-parity is violated}

In this case the $\widetilde{s b}_{1}$ pair so produced will decay via the R-parity violating terms $\lambda^{\prime} L Q D^{c}$ or $\lambda^{\prime \prime} U^{c} D^{c} D^{c}$ in the superpotential. In general, $\lambda^{\prime}$ and $\lambda^{\prime \prime}$ couplings are not considered simultaneously, otherwise it will lead to unwanted baryon decay. Since nonzero $\lambda^{\prime \prime}$ couplings would give only multi-jets in the final state, which would likely be buried under QCD backgrounds, we only consider the $\lambda^{\prime}$ coupling in the following.

By the right-handed nature of the strange-beauty squark in our scenario, the third index in the $\lambda^{\prime}$ coupling is either 2 or 3 , and we only consider $\lambda_{i i 3}^{\prime}, \lambda_{i i 2}^{\prime}$ with $i=1,2$. The strangebeauty squark will decay into $e^{-} u$ or $\mu^{-} c$. Therefore, the strange-beauty squark behaves like scalar leptoquarks of the first or second generation, respectively. The decay mode of $\tau^{-} t$ is not feasible at the Tevatron. The current published limits [16] from CDF are $213 \mathrm{GeV}$ and $202 \mathrm{GeV}$ for the first and second generation leptoquarks while $\mathrm{D} \emptyset$ obtained limits of 225 and $200 \mathrm{GeV}$, respectively. The latest preliminary limits [16] from CDF are 230 and $240 \mathrm{GeV}$, respectively, while those from $\mathrm{D} \emptyset$ are 231 and $186 \mathrm{GeV}$, respectively. In one of the preliminary plots, the combined limits from all $\mathrm{CDF}$ and $\mathrm{D} \emptyset$ run I and II data can push the first generation leptoquark limit to around $260 \mathrm{GeV}$, which is very impressive. ${ }^{4}$ The sensitivity reach in run II has been studied

\footnotetext{
${ }^{4}$ These limits are for the leptoquarks that decay entirely into charged leptons and quarks. If the leptoquark also decays into a neutrino and a quark, the corresponding limit is somewhat weaker.
} 
TABLE II. Cross sections in fb for direct squark-pair production at the Tevatron with $\sqrt{s}=1.96 \mathrm{TeV}$, for $0,1,2 b$-tagged events. The imposed cuts are $p_{T j}>15 \mathrm{GeV},\left|\eta_{j}\right|<2$, and $p_{T}>40 \mathrm{GeV}, b$-tagging efficiency $\epsilon_{b t a g}=0.6$, and a mistag probability of $\epsilon_{m i s}=0.0$. In parentheses, we give the contribution fed down from direct gluino-pair production.

\begin{tabular}{|c|c|c|c|c|c|c|}
\hline$m_{\widetilde{s b}_{1}}(\mathrm{GeV})$ & $0 b$-tag & $1 b$-tag & $2 b$-tag & $0 b$-tag & $1 b$-tag & $2 b$-tag \\
\hline & & $\sin ^{2} \theta_{m}=1$ & & & $\sin ^{2} \theta_{m}=0.75$ & \\
\hline 150 & $115(0.11)$ & $288(0.54)$ & $175(2.2)$ & $190(0.29)$ & $284(0.89)$ & 104 (1.6) \\
\hline 200 & $26(0.091)$ & $70(0.49)$ & $47(2.2)$ & $44(0.27)$ & $70(0.85)$ & $28(1.7)$ \\
\hline 250 & $6.1(0.090)$ & $17(0.49)$ & $11(2.2)$ & $11(0.27)$ & $17(0.85)$ & $6.8(1.7)$ \\
\hline 300 & $1.5(0.090)$ & $4.2(0.49)$ & $2.9(2.2)$ & $2.6(0.27)$ & $4.2(0.85)$ & $1.7(1.7)$ \\
\hline 350 & $0.38(0.090)$ & $1.1(0.49)$ & $0.72(2.2)$ & $0.66(0.27)$ & $1.1(0.86)$ & $0.43(1.7)$ \\
\hline 400 & $0.094(0.090)$ & $0.26(0.49)$ & $0.18(2.2)$ & $0.16(0.27)$ & $0.26(0.86)$ & $0.11(1.7)$ \\
\hline \multirow[t]{2}{*}{450} & $0.022(0.096)$ & $0.06(0.51)$ & $0.04(2.2)$ & $0.038(0.28)$ & $0.061(0.87)$ & $0.025(1.7)$ \\
\hline & & $\sin ^{2} \theta_{m}=0.5$ & & & $\sin ^{2} \theta_{m}=0.25$ & \\
\hline 150 & $283(0.66)$ & $243(1.2)$ & $51(1.0)$ & $395(1.3)$ & $165(1.1)$ & $17(0.40)$ \\
\hline 200 & $68(0.63)$ & $61(1.1)$ & $14(1.0)$ & $96(1.3)$ & $42(1.1)$ & $4.6(0.42)$ \\
\hline 250 & $16(0.62)$ & $15(1.1)$ & $3.3(1.0)$ & $23(1.3)$ & $10(1.1)$ & $1.1(0.42)$ \\
\hline 300 & $4.0(0.63)$ & $3.7(1.1)$ & $0.84(1.0)$ & $5.8(1.3)$ & $2.5(1.1)$ & $0.28(0.42)$ \\
\hline 350 & $1.0(0.63)$ & $0.93(1.1)$ & $0.21(1.0)$ & $1.4(1.3)$ & $0.64(1.1)$ & $0.071(0.43)$ \\
\hline 400 & $0.25(0.63)$ & $0.23(1.2)$ & $0.052(1.1)$ & $0.35(1.3)$ & $0.16(1.1)$ & $0.017(0.43)$ \\
\hline 450 & $0.058(0.64)$ & $0.053(1.2)$ & $0.012(1.0)$ & $0.083(1.3)$ & $0.037(1.1)$ & $0.004(0.42)$ \\
\hline
\end{tabular}

in TeV2000 report [17]. The reach on the first or second generation leptoquarks are 235 and $325 \mathrm{GeV}$ with a luminosity of 1 and $10 \mathrm{fb}^{-1}$, respectively. Apparently, the preliminary limits obtained by $\mathrm{CDF}$ and $\mathrm{D} \emptyset$ with a luminosity of $\sim 200 \mathrm{pb}^{-1}$ are already very close to or even surpass the sensitivity reach quoted in TeV2000 report. Therefore, we believe that the limit that can be reached at the end of run II $\left(2 \mathrm{fb}^{-1}\right.$ ) is very likely above $300 \mathrm{GeV}$. With an order more luminosity, the limit may be able to reach $350 \mathrm{GeV}$ : see the total cross section in Fig. 3(a).

\section{C. $\widetilde{s b}_{1}$ is the NLSP}

In this case the $\widetilde{s b}_{1}$ so produced will decay into a strange or beauty quark plus the neutralino in the supergravity framework or the gravitino (or via an intermediate neutralino into a photon and a gravitino) in the gauge-mediated framework. Experimentally, the signature is similar, except for the fact that the neutralino is of order $100 \mathrm{GeV}$ while the gravitino is virtually massless compared to the collider energy. We simplify the picture by modelling the decay as $\widetilde{s b}$ $\rightarrow s / b \tilde{\chi}_{1}^{0}$ and by varying the mass of the neutralino. In addition, we have to check if the strange-beauty squark will decay within the detector. In the SUGRA case, the decay rate is of electroweak strength hence the decay is prompt. However, in the gauge-mediated case, the decay rate scales as $\sim 1 / F_{\text {SUSY }}^{2}$, where $\sqrt{F_{\text {SUSY }}}$ is the dynamical SUSY breaking scale. Therefore, if $\sqrt{F_{\text {SUSY }}}$ is so large, the strange-beauty squark behaves like a stable particle inside the detector. Reference [15] showed that, for $\sqrt{F_{\text {SUSY }}} \gtrsim 10^{7} \mathrm{GeV}$ the scalar tau NLSP would behave like a stable particle inside a typical particle detector. This value applies to the strange-beauty squark as well, up to a color factor. If it is stable, one goes back to case (i). So here we focus on the prompt decay of the strange-beauty squark, which is considered to be the more popular case.

There are 2 quark jets in the final state of $\widetilde{s b}_{1} \widetilde{s b}_{1}^{*}$ pair production, each of them either strange or beauty flavored, and with large missing energy due to the neutralinos or gravitinos. We impose the following cuts on the jets and missing transverse momentum, and we choose the following $b$-tagging and mistag efficiencies ${ }^{5}$

$$
\begin{gathered}
p_{T j}>15 \mathrm{GeV}, \quad\left|\eta_{j}\right|<2.0, \quad p_{T}>40 \mathrm{GeV}, \\
\epsilon_{\text {btag }}=0.6, \quad \epsilon_{\text {mis }}=0.05 .
\end{gathered}
$$

Note that the branching ratio of the strange-beauty squark into a $b$ quark scales as $\sin ^{2} \theta_{m}$.

We have tested our parton-level Monte Carlo program as follows. Most events generated pass the jet $\left(p_{T j}\right.$ and $\left.\left|\eta_{j}\right|\right)$ requirements, as long as the mass difference between the $\widetilde{s b}_{1}$ and $\tilde{\chi}_{1}^{0}$ is larger than $50 \mathrm{GeV}$. Taking $B\left(\widetilde{s b}_{1} \rightarrow b \widetilde{\chi}_{1}^{0}\right)=1$ (a standard $\tilde{b}$ squark), we verify our input $b$-tagging efficiency, i.e. the ratio of $0: 1: 2 b$-tagged jets is 16:48:36. Choosing the $B\left(\widetilde{s b}_{1} \rightarrow b \widetilde{\chi}_{1}^{0}\right)=0.5$ value expected in our scenario, the ratio of $0: 1: 2 \quad b$-tagged jets becomes 49:42:9. The doubletag approach becomes far less effective, but if we only require at least one $b$-tagged jet in the final state, the overall efficiency is about 0.5. On one hand, this is a dilution compared to the standard $\widetilde{b}$ squark pair production, which gives overall efficiency of 0.84 . On the other hand, the prospect is still very good for run II.

\footnotetext{
${ }^{5}$ The mistag efficiency is the probability that a non- $b$ jet is detected as a $b$-jet.
} 
In Table II, we show the cross sections in units of $\mathrm{fb}$ for direct squark-pair production, with the squark decaying into either $s / b$ plus a neutralino at the Tevatron with $\sqrt{s}$ $=1.96 \mathrm{TeV}$. We have set $m_{\tilde{\chi}_{1}^{0}}=100 \mathrm{GeV}$; other values of $m \tilde{\chi}_{1}^{0}$ do not affect the result in any significant way, so long as the mass difference between the squark and neutralino is larger than about $50 \mathrm{GeV}$. Note that the production cross section itself is almost independent of $\sin ^{2} \theta_{m}$ and $m_{\tilde{g}}$. This is because the dominant production channel is the standard QCD $s$-channel $q \bar{q} \rightarrow \widetilde{s b_{1}} \widetilde{s b}_{1}^{*}$ process and we have imposed $m_{\tilde{g}} \gtrsim 500 \mathrm{GeV}$. The gluino-pair production process, which is also independent of $\sin ^{2} \theta_{m}$, is only $2.9 \mathrm{fb}$, and only becomes relevant for $m_{\widetilde{s b}} \geq 300 \mathrm{GeV}$. The branching ratio of the $\widetilde{s b}_{1}$ into a $b$ quark, however, scales as $\sin ^{2} \theta_{m}$. We therefore give results for $\sin ^{2} \theta_{m}=1,0.75,0.5,0.25$, and for 0,1 , and 2 $b$-tagged jet events. The case for $\sin ^{2} \theta_{m}=1$ is the same as a standard $\tilde{b}$ squark.

We see that, for $\sin ^{2} \theta_{m} \gtrsim 0.5$, if we only require at least one $b$-tagged jet rather than demanding double-tag, the cross section does not change drastically as $\sin ^{2} \theta_{m}$ decreases from 1 to 0.5 . Requiring a minimum of 10 signal events ${ }^{6}$ as suggestive evidence for such a squark, with an integrated luminosity of $2 \mathrm{fb}^{-1}$ the sensitivity is around $300 \mathrm{GeV}$, if $\sin ^{2} \theta_{m} \geq 0.5$. If the integrated luminosity can go up to $20 \mathrm{fb}^{-1}$, then the sensitivity increases to $350 \mathrm{GeV}$.

We emphasize that the double-tag vs single-tag ratio contains information on $\sin ^{2} \theta_{m}$, while their sum, when compared with the standard $\tilde{b}$ squark pair production, provides additional consistency check on cross section vs mass. Such work would depend on more detailed knowledge of the detector, which we leave to the experimental groups.

\section{DISCUSSION AND CONCLUSION}

In this work, we have considered the SUSY scenario that the only light degrees of freedom are the right-handed strange-beauty squark $\left(m_{\widetilde{s b}_{1}} \gtrsim 200 \mathrm{GeV}\right)$ and gluino $\left(m_{\tilde{g}}\right.$ $=500 \mathrm{GeV})$. Such a light squark is a result of a nearmaximal mixing in the 2-3 sector of the right-handed squarks, which is in turn a result of approximate Abelian flavor symmetry.

We have performed calculations for direct strange-beauty squark-pair production, as well as the feed down from gluino-pair production and the associated production of $\widetilde{s b}_{1}$ with gluino. It turns out that the dominant contribution comes from direct squark-pair production as long as the

${ }^{6}$ From an experimental study of scalar bottom quark search by $\mathrm{CDF}$ [18], the background comes from $t \bar{t}$, single and pair vector boson production. Using $b$-tagging the number of background events was reduced to the few events level with run I data. Therefore, with ten times more luminosity in run II but much improved $b$-tagging and event selection techniques, the background should be of $\mathcal{O}(10)$ events. If so, 10 signal events above this background would have a sensitivity of 2 sigma or more. So, for simplicity we use 10 signal events as a rough criteria for sensitivity reach. squark mass is below $300 \mathrm{GeV}$. As one has to require $m_{\tilde{g}}$ $\gtrsim 500 \mathrm{GeV}$, which comes from low energy bounds, gluino pair production is in general subdominant. However, as the squark mass is above $300 \mathrm{GeV}$, the feed down from gluinopair production with $m_{g}=500 \mathrm{GeV}$ becomes sizable. Furthermore, many new avenues such as $s \bar{s}(s s) \rightarrow \widetilde{s b}_{1} \widetilde{s b}_{1}^{(*)}$ opens up. These are, however, very suppressed at Tevatron energies because of heavy gluino mass.

We have studied three decay scenarios of the strangebeauty squarks that are relevant for the search at the Tevatron, which is of immediate interest because it can be readily done in the near future. The three decay modes that we have considered are (i) (quasi)stable $\widetilde{s b}_{1}$ as in $\widetilde{s b}_{1}$-LSP SUSY or in gauge-mediated SUSY breaking with a very large $\sqrt{F}$, (ii) $R$-parity violating decay of $\widetilde{s b}_{1}$ (hence $\widetilde{s b}_{1}$ behaves like a leptoquark), and (iii) the popular case of $\widetilde{s b}_{1} \rightarrow s / b \widetilde{\chi}_{1}^{0}$ decay, where $\tilde{\chi}_{1}^{0}$ is the LSP. In the first case, the $\widetilde{s b}_{1}$ once produced would hadronize into a massive stable charged particle like a "heavy muon," which would ionize and form a track in the central tracking system and in the outer muon chamber. This is a very clean signature. The sensitivity for run II with an integrated luminosity of $2 \mathrm{fb}^{-1}$ is up to about $300 \mathrm{GeV}$, which may increase to about $350 \mathrm{GeV}$ with an order more luminosity. In the second case, the $\widetilde{s b}$ decays like a leptoquark of the first or second generation. The best current limit is $260 \mathrm{GeV}$ (preliminary [16]) for the first generation. This is already at the sensitivity level of the Tev2000 study [17] for $2 \mathrm{fb}^{-1}$. With an order more luminosity, the limit should reach $350 \mathrm{GeV}$. In the last case, $\widetilde{s b}_{1} \rightarrow s / b \widetilde{\chi}_{1}^{0}$ decay leads to multiple $b$-jets plus large missing energy in the final state. The number of $b$-tag events depends on the mixing angle $\sin \theta_{m}$, because the branching ratio of $\widetilde{s b}_{1} \rightarrow b \widetilde{\chi}_{1}^{0}$ scales as $\sin ^{2} \theta_{m}$. As long as $\sin ^{2} \theta_{m} \gtrsim 0.5$, the sensitivity at the run II with $2 \mathrm{fb}^{-1}$ goes up to about $300 \mathrm{GeV}$. With improved $b$-tagging in run II, one can also make use of the single versus double $b$-tag ratio as well as the $b$-tagged cross section to determine $m_{\widetilde{s b}_{1}}$ and the mixing angle $\sin ^{2} \theta_{m}$.

At the LHC $\widetilde{s b} \widetilde{s b}_{1}^{*}$ and $\tilde{g} \tilde{g}$ pair production cross sections are comparable, with $g g$ fusion being the dominant mechanism. Unlike at the Tevatron, the associated production of $s g \rightarrow \widetilde{s b}_{1} \tilde{g}$ becomes interesting at the LHC. Nevertheless, $\widetilde{s b}_{1} \widetilde{s b}_{1}$ or $\widetilde{s b}_{1}^{*} \widetilde{s b}_{1}^{*}$ pair production remains relatively unimportant. With $\widetilde{s b}_{1}$ as light as $200 \mathrm{GeV}, \widetilde{s b}_{1} \widetilde{s b}_{1}^{*}$ pair production may be relatively forward. On the other hand, $\tilde{g} \widetilde{g}$ events, followed by $\widetilde{g} \rightarrow \widetilde{s b}_{1} \bar{s} / \bar{b}$, would have extra hard jets to provide more handles. The $\widetilde{s b}_{1} \tilde{g}$ final state, if it can be separated, can probe the mixing angle $\cos ^{2} \theta_{m}$ in the production cross section. Discovery of the strange-beauty squark at the LHC should be no problem at all, but the richness demands a more dedicated study, which we leave for future work.

In conclusion, the recent possible $C P$ violation discrepancy in $B \rightarrow \phi K_{S}$ decay suggests the possibility of a light strange-beauty squark $\widetilde{s b}_{1}$ that carries both strange and beauty flavors. Such an unusual squark can be searched for at 
the Tevatron run II, with the precaution that $\widetilde{s b}_{1}$ can decay into a beauty or strange quark, and the standard $\widetilde{b}$ search should be broadened. Discovery up to $300 \mathrm{GeV}$ is not a problem, and anomalous behavior in both production cross sections and the single versus double tag ratio may provide confirming evidence for the strange-beauty squark.

\section{ACKNOWLEDGMENT}

This research was supported in part by the National Science Council of Taiwan R.O.C. under grant no. NSC 922112-M-007-053- and NSC-92-2112-M-002-024, and by the MOE CosPA project.
[1] Y. Nir and N. Seiberg, Phys. Lett. B 309, 337 (1993); M. Leurer, Y. Nir, and N. Seiberg, Nucl. Phys. B420, 468 (1994).

[2] C.K. Chua and W.S. Hou, Phys. Rev. Lett. 86, 2728 (2001).

[3] A. Arhrib, C.K. Chua, and W.S. Hou, Phys. Rev. D 65, 017701 (2002).

[4] Belle Collaboration, K. Abe et al., Phys. Rev. D 67, 031102 (2003)

[5] Belle Collaboration, K. Abe et al., Phys. Rev. Lett. 91, 261602 (2003).

[6] T. Browder, Int. J. Mod. Phys. A 19, 965 (2004).

[7] C.K. Chua, W.S. Hou, and M. Nagashima, Phys. Rev. Lett. 92, 201803 (2004).

[8] CDF Collaboration, T. Affolder et al., Phys. Rev. Lett. 88, 041801 (2002); "Higgs and Supersymmetry at collider experiments," talk by M. Schmitt at the Lepton-Photon Symposium 2003, Fermilab, U.S.A.

[9] W. Beenakker, R. Hopker, M. Spira, and P. Zerwas, Nucl. Phys. B492, 51 (1997); hep-ph/9611232.

[10] Particle Data Group, K. Hagawara et al., Phys. Rev. D 66, 010001 (2002).

[11] CDF Collaboration, F. Abe et al., Phys. Rev. D 46, R1889
(1992); CDF Collaboration, A. Connolly, hep-ex/9904010; CDF Collaboration, K. Hoffman, hep-ex/9712032.

[12] CDF Collaboration, D. Acosta et al., Phys. Rev. Lett. 90, 131801 (2003).

[13] H. Baer, K. Cheung, and J. Gunion, Phys. Rev. D 59, 075002 (1999).

[14] K. Cheung and G. Cho, Phys. Rev. D 67, 075003 (2003); 69, 017702 (2004).

[15] J. Feng and T. Morii, Phys. Rev. D 58, 035001 (1998).

[16] CDF Collaboration, F. Abe et al., Phys. Rev. Lett. 79, 4327 (1997); 81, 4806 (1998). The latest preliminary results from $\mathrm{CDF}$ is available at http://www-cdf.fnal.gov/physics/exotic/ exotic.html; DØ Collaboration, V.M. Abazov et al., Phys. Rev. D 64, 092004 (2001); B. Abbott et al., Phys. Rev. Lett. 84, 2088 (2000). The latest preliminary results from DФ is available at http://www-d0.fnal.gov/Run2Physics/np/

[17] D. Amidei et al., "Future Electroweak Physics at the Fermilab Tevatron," Report of the tev_2000 Study Group, FermilabPub-96/082, 1996.

[18] CDF Collaboration, T. Affolder et al., Phys. Rev. Lett. 84, 5704 (2000). 